\title{
Comparación de competencias parentales en padres y en madres con hijos e hijas adolescentes
}

\author{
Raquel Amaya Martínez-González \\ Universidad de Oviedo, España \\ mail: ramayaduniovi.es \\ ORCID: https://orcid.org/0000-0003-3254-8655
}

\section{Beatriz Rodríguez-Ruiz}

Universidad de Oviedo, España

mail: rodriguezbeatriz@uniovi.es

ORCID: https://orcid.org/0000-0002-3009-6766

\author{
María Teresa Iglesias García \\ Universidad de Oviedo, España \\ mail: teresaiduniovi.es \\ ORCID: https://orcid.org/0000-0002-9577-7693
}

\section{RESUMEN}

El rol parental con adolescentes requiere de los progenitores competencias personales emocionales y sociales. Hay pocos estudios de género que analicen estas competencias en el padre y en la madre en la misma unidad familiar. El objetivo de este estudio es identificar si estas competencias difieren en ambos progenitores, y si varían también en función de su nivel de estudios y de la fase inicial o tardía de la adolescencia. Han participado 752 madres y 670 padres. Se aplicó la Escala de Competencias Parentales Emocionales y Sociales para progenitores de Adolescentes (ECOPES-A), analizando cuatro factores: Control-Relajación, Autoestima, Imposición y Comunicación. A través de $t$ de Student y Análisis de Varianza los resultados indican mayor competencia comunicativa en las madres que en los padres para relacionarse con sus adolescentes, y para fomentar su autoestima; las madres, sin embargo, se autoevalúan con menor habilidad que los padres en autocontrol emocional. Las competencias parentales son más altas cuando el nivel educativo de ambos también lo es, salvo en autoestima. Ambos progenitores tienden a ser más impositivos con sus adolescentes más jóvenes. Entre las implicaciones del estudio se destaca reforzar la gestión emocional de los progenitores a través de programas de parentalidad positiva.

Palabras Clave: Competencias parentales, Adolescentes, Género, Nivel Educativo.

\section{Comparison of parental competences in fathers and mothers with adolescent children}

\section{ABSTRACT}

Parenting adolescents requires personal, emotional and social competencies from the parents. There are few gender studies that analyze these competencies in the father and the mother in the same family. The objective of this study is to identify if these competencies differ in both parents, and if they also vary according to their educational level and the stage of early or late adolescence. 752 mothers and 670 fathers participated. The Emotional and Social Parenting Competencies Scale for Parents of Adolescents (ECOPES-A) was applied, analyzing four factors: Control-Relaxation, Self-esteem, Imposition, and Communication. Through Student's $t$ test and Analysis of Variance, the results indicate greater communicative competence in mothers than in fathers to interact with their adolescents, and to promote their self-esteem; mothers, however, evaluate themselves with less ability than fathers in emotional self-control. Parental competencies are higher when the educational level of both is also higher, except in self-esteem. Both parents tend to be more imposing with their younger teens. Among the implications of the study is to strengthen both parent's self-emotional management through positive parenting programs.

Keywords: Parenting Competences, Adolescence, Gender, Educational level.

ISSN: 0210-2773

DOI: https://doi.org/10.17811/rifie.50.4.2021.777-786 


\section{Introducción}

Educar adolescentes supone un reto emocional para muchos padres y madres, como ejemplifica el siguiente comentario: " $m i$ adolescente pasa de una postura negativa o apática a un optimismo sin razón aparente. Estos cambios me hacen dudar, me descontrolan,..." (Martínez et al., 2007, p. 34). Tradicionalmente se ha considerado que la relación entre adolescentes y progenitores es distante por su búsqueda de autonomía, por su cuestionamiento de normas familiares y sociales (García-Linaresa, et al., 2014; Sanders, 2013), y por las conductas de riesgo que pueden asumir, generando situaciones estresantes en la convivencia familiar (Martínez-González et al., 2007). Las teorías actuales sobre el desarrollo evolutivo aportan una visión más positiva de la adolescencia, enfatizando su potencial de cambio a través de las relaciones mutuamente influyentes entre la persona y los sistemas culturales y ecológicos en los que interactúa, entre los que se encuentra la familia (Bronfenbrenner, 1987). Desde la teoría del Desarrollo Positivo del Adolescente (DPA) (Lerner et al., 2005) se entiende que es posible lograr un desarrollo adaptativo de los jóvenes si las interacciones que establecen en sus contextos tienden a buscar el bienestar de todas las partes. Este enfoque se focaliza en la promoción de sus competencias personales y de una interacción positiva en el entorno, a diferencia del enfoque tradicional centrado fundamentalmente en la reparación de problemas y en la prevención de conductas de riesgo. En este cometido, la familia juega un papel clave como contexto emocional y afectivo y como principal agente socializador; para ello, los padres y madres han de contar con competencias que les faciliten interaccionar con sus adolescentes de modo positivo, con un estilo educativo democrático basado en el respeto y en el entendimiento mutuo.

\section{Competencias Parentales}

La calidad con que los progenitores afrontan las relaciones con sus adolescentes está condicionada, entre otros factores, por sus competencias parentales; es decir, por el conjunto de capacidades, habilidades y actitudes que tienen para educar a sus hijos e hijas en base a las necesidades que estos/as presenten, a la etapa evolutiva en que se encuentren y a las circunstancias familiares (Masten y Curtis, 2000; Waters y Sroufe, 1983). Las competencias parentales facilitan a los adultos organizar la vida familiar, desarrollar estilos educativos positivos y promover el desarrollo integral de sus hijos e hijas (Martínez-González, 2009; Ponzetti, 2016; Rodrigo et al., 2015), en línea con lo que establece la teoría DPA (Lerner et al., 2003). A este respecto, la teoría de los Estilos Parentales (Baumrind, 1991a; Maccoby y Martin, 1983) y la Teoría de la Autodeterminación (Ryan y Deci, 2017), indican que los adolescentes expuestos a un estilo de comunicación parental asertivo, no impositivo, que refleje equilibrio emocional y autoestima parental presentan mayor madurez, autonomía de pensamiento y de acción, y conductas más constructivas y adaptadas socialmente que aquellos que interactúan bajo patrones de comunicación parental impulsivos e impositivos. De acuerdo con Bonds et al. (2002), mantener un estilo educativo positivo depende mucho del nivel de estrés parental, dado que este incrementa la impulsividad y disminuye la autorregulación emocional y del comportamiento. Por tanto, la habilidad de los progenitores para gestionar sus emociones constituye una competencia relevante por su potencialidad para facilitar una relación constructiva con sus adolescentes que contribuya a su desarrollo positivo (Garaigordobil, 2005; Pons-Salvador et al., 2005). A este respecto cabe preguntarse si estas competencias se manifiestan por igual en los progenitores en función de su género, nivel educativo y de la fase inicial o tardía de la adolescencia de sus hijos e hijas.

\section{Género, nivel educativo, fase adolescente y competencias pa- rentales}

La mayoría de los estudios realizados sobre estilos educativos y competencias parentales analizan conjuntamente al padre y a la madre (Kerr y Stattin, 2000; Sullivan, 2004; Wang et al., 2011). Sin embargo, surge el interrogante de si estas competencias varían en ambos, y si estas variaciones producen efectos diferenciales en los adolescentes. Baumrind (1991b) encontró que un $76 \%$ de parejas coincidían en su estilo parental; por su parte, Fletcher et al. (1999) hallaron que los adolescentes que tenían al menos un progenitor con estilo educativo democrático mostraban mayor ajuste personal y social que quienes no tenían ninguno. Algunos estudios desarrollados desde la teoría DPA indican que el estilo de la madre es más afectuoso y comunicativo que el del padre, y pronostica mejor el ajuste psicosocial de los adolescentes, facilitando su autorregulación emocional (Laible y Caro, 2004; Sánchez et al., 2008).

Actualmente en muchas familias trabajan ambos progenitores fuera del hogar, necesitando ambos involucrarse en la crianza y educación de sus hijos e hijas. Sin embargo, no lleva necesariamente asociado que las madres disminuyan su implicación (Meteyer y Perry-Jenkins, 2010), tal como sugiere la teoría sobre el rol de género (Potuchek, 1990). Moon y Hoffman (2008) encontraron que la dedicación de las madres respecto al cuidado físico y al apoyo emocional era mayor que la de los padres, y Pleck (1997) identificó que los padres realizan, sobre todo, actividades de ocio y que su implicación es mayor cuando los hijos e hijas son pequeños/as que cuando son adolescentes. Por su parte, en la revisión realizada por Yárnoz Yaben (2006) con respecto a los cambios sociales de las últimas décadas y su influencia en la distribución de funciones del padre y de la madre, se concluye que la valoración que hacen los propios adolescentes sobre la cercanía y calidad de la relación con el padre correlaciona positivamente con sus resultados académicos, y negativamente con la conducta sexual promiscua, robo, o adicciones, enfatizando así la contribución del padre al bienestar emocional de sus adolescentes.

Otros estudios indican que las conductas de riesgo en adolescentes son más frecuentes en la fase inicial de esta etapa evolutiva, y cuando proceden de familias con bajo nivel socioeconómico y cultural (Sullivan et al., 2004; Wang et al., 2011). Kan y Tsai (2005) encontraron que el estilo educativo parental actúa como variable intermediaria entre el estatus socioeducativo, el rendimiento escolar de los hijos e hijas y su estado de salud mental. McLoyd y Smith (2002) y Belsky et al. (2007) obtuvieron que los progenitores con nivel socioeducativo bajo mostraban más indicadores de baja competencia parental que quienes tenían niveles educativos más altos, por su mayor tendencia hacia el estilo autoritario, que afecta negativamente al desarrollo de la autonomía y de la autoestima en los hijo/as; derivando en un menor logro académico por su parte. De igual modo, Huang (2018) encontró que mientras los estilos parentales democrático y permisivo se identifican más en familias con nivel socioeducativo alto, los estilos autoritario y negligente se asocian más con niveles socioeducativos bajos. Tomando en cuenta, además, el género de los progenitores, Bøe et al. (2014) encontraron que las madres con mayor nivel educativo tendían a emplear poco con sus hijos e hijas métodos de disciplina basados en gritos o amenazas, en comparación con las madres con menos estudios, que empleaban 
más estos métodos de control; como efecto de estos últimos, los hijos e hijas mostraban mayores índices de conductas agresivas, hiperactividad, ansiedad y depresión.

A pesar de los estudios consultados, es aún escaso el conocimiento sobre las competencias parentales diferenciales por género de ambos progenitores, y sobre cómo se manifiestan en función de su nivel de estudios y de la fase inicial o tardía de la adolescencia de sus hijos e hijas. El presente trabajo tiene como objetivo profundizar en estos aspectos con la finalidad de identificar posibles implicaciones para la intervención familiar.

\section{Método}

\section{Participantes}

El estudio incluye 1422 participantes distribuidos en 752 madres y 670 padres (80\% parejas de la misma unidad familiar) con adolescentes. Para su selección se efectuó un muestreo aleatorio estratificado por edades y municipio de residencia en Asturias (España) tomando como población 51936 familias con hijos e hijas entre 12 y 18 años (INEBase). Se asumió un 5\% de error y un nivel de confianza del $99 \%$, resultando un mínimo necesario de 656 participantes. La muestra obtenida tiene mayoritariamente entre 42 y 53 años (74,1\% padres; 74,9\% madres); su tipología familiar predominante es biparental $(79,8 \%$ madres; $86,3 \%$ padres), siendo escasas las familias monoparentales (9,4\% madres; $2,6 \%$ padres), reconstituidas (3,1\% madres; $2,9 \%$ padres) y extensas (3,1\% madres; $3,2 \%$ padres). La mayoría trabaja fuera del hogar (84,8\% padres; $67,8 \%$ madres). Su nivel de estudios se distribuye en universitario (39,8\% madres; $33,1 \%$ padres), bachillerato/FP (36,9\% madres; $40,9 \%$ padres) y obligatorio $(23,3 \%$ madres; $26,0 \%$ padres). Sus adolescentes cursan estudios en centros públicos $(71,3 \%)$, concertados $(16,8 \%)$ o privados $(11,9 \%)$, ubicados en áreas rurales $(36,1 \%)$ o urbanas (63,9\%). Aquellos/as que se encuentran en la etapa inicial de la adolescencia (12-16 años) cursan educación secundaria obligatoria (ESO) (64,8\%) y quienes están en la etapa más tardía de este estadio evolutivo estudian educación secundaria postobligatoria (bachiller/formación profesional, B/FP) $(35,2 \%)$.

\section{Instrumento y procedimiento}

Se aplicó la Escala de Competencias Parentales Emocionales y Sociales para progenitores de Adolescentes (ECOPES-A) validada por las autoras de este trabajo, que consta de 12 ítems con respuesta Likert de cuatro alternativas (1Nunca hasta 4Siempre). Están distribuidos en cuatro factores que explican el 46,26\% de la varianza tras realizar un proceso de validación cruzada (Browne y Cudeck, 1993) con análisis factorial exploratorio (AFE) y confirmatorio (AFC) (chi cuadrado significativo, $\chi 2=109.359$ (48), $p<.000 ; C M I N / D F=2.278 ; R M S E A=0.042 ; S R M R=0.039 ; G F I$ $=0.976 ; T L I=0.960 ; C F I=0.971)$. Los factores son: F1-Control y Relajación $(\alpha$ Cronbach $=.80)$, con tres ítems: 1$)$ Sé cómo relajarme y controlar mis emociones; 2) En el día a día me relajo y controlo cuando estoy alterado/a; 3) Sé relajarme y controlar mis emociones ante mis hijos/as; F2-Autoestima $(\alpha$ Cronbach $=$ .70), con tres ítems: 1) Tengo buena opinión de mí mismo/a sobre cómo educo a mis hijos/as; 2) Creo que soy un buen padre/ madre; 3) Tengo buena opinión de mí mismo/a; F3-Imposición $(\alpha$ Cronbach $=.62)$, con tres ítems: 1$)$ Cuando mis hijos/as me desobedecen lo mejor es imponerse para solucionar la situación; 2) Cuando surge un conflicto con mis hijos/as, les digo yo lo que hay que hacer y se termina el problema; y 3) Cuando mis hijos/as intentan salirse con la suya para conseguir algo, yo me impongo más para controlar la situación; y F4-Comunicación ( $\alpha$ Cronbach $=.63$ ), con tres ítems: 1) Les digo a mis hijos/as cómo me siento con su manera de actuar; 2) En el día a día suelo decirles a mis hijos/as lo positivo que veo en ellos/as, y 3) Hago ver a mis hijos/ as que son capaces de tomar decisiones. El índice de fiabilidad alpha de Cronbach $(\alpha)$ total de la escala resultó .714. La escala se acompañó de los ítems sociodemográficos género, nivel de estudios de los progenitores y etapa escolar de los y las adolescentes, que se analizan en este trabajo.

El acceso a las familias se realizó a través de centros escolares, que colaboraron tras firmar su consentimiento, entregando a los hijos/as un sobre con dos cuestionarios; uno para la madre y otro para el padre, y una carta de presentación. Se distribuyeron 1968 sobres en centros públicos, concertados y privados según la proporción que representaban a nivel poblacional. Los escolares devolvieron 1422 cuestionarios (752 de madres y 670 de padres), lo que permitió superar el tamaño de muestra representativo previsto (656 participantes).

\section{Análisis de datos}

Los contrastes sobre competencias parentales en función del género de los progenitores, de su nivel de estudios (obligatorios, secundarios o universitarios) y de la etapa escolar de los adolescentes (ESO o bachiller/FP) se realizaron tanto en los factores como en sus ítems o indicadores con $t$ de Student y análisis de varianza. El tamaño del efecto en los contrastes de dos grupos se estimó con $d$ de Cohen, interpretando su magnitud como baja cuando $0<d<.20$; media-moderada cuando $.20 \leq d \leq .50$; y alta cuando $d>.50$ (Cohen, 1988); en los contrastes de tres grupos, se comprobó con eta cuadrado $\left(\eta^{2}\right)$, interpretando la magnitud pequeña si $\eta^{2}<06$, moderada cuando $.06 \leq \eta^{2} \leq .14$, y grande con $\eta^{2} \geq .14$ (Cohen, 1973). Se estimó también con el modelo lineal general univariante el efecto de la interacción entre el género de los progenitores y su nivel de estudios sobre las competencias parentales analizadas en los factores. Los cálculos se efectuaron con SPSS 22.0.

\section{Resultados}

Competencias parentales en función del género de los progenitores

Los resultados descriptivos indican que, en general, tanto los padres como las madres se perciben con autoestima alta, tanto en lo que respecta a su persona, como a su rol parental (F2-Autoestima, $M=3.09 ; S x=.585)$, y con habilidades para comunicarse positivamente con sus adolescentes (F4-Comunicación, $M=3.11$; $S x=.636)$. Sin embargo, también se detectan valores relevantes en imposición (F3-Imposición, $M=2.36$; $S x=.662$ ), y falta de control emocional (F1-Control-Relajación, $M=2.73$; $S x=.622$ ). El contraste de grupos por género indica diferencias significativas en F1-Control-Relajación ( $p=.000, d=.25)$, identificando mayor regulación emocional en los padres que en las madres, con un tamaño del efecto medio. También se obtienen diferencias en F4-Comunicación ( $p=.000, d=.46)$, detectándose mayor capacidad en las madres que en los padres, con un tamaño del efecto medio. Cuando el análisis se realiza con cada ítem como indicador de estos factores (Tabla 1) se confirman puntuaciones significativamente mayores en los padres que en las madres en todos los indicadores de F1-Control-Relajación; sin embargo, las madres obtienen una puntación significativamente mayor que los padres en todos los indicadores de F4-Comunicación; en ambos factores con tamaño del efecto medio. 
Tabla 1

Diferencias significativas en función del género (madre o padre)

\begin{tabular}{|c|c|c|c|c|c|c|}
\hline Factor & Indicador/Ítem & Fuente & $M(D T)$ & $t$ & $p$ & $d$ \\
\hline \multirow{6}{*}{$\begin{array}{c}\text { F1- } \\
\text { Control } \\
\text { y } \\
\text { Relajación }\end{array}$} & \multirow{2}{*}{$\begin{array}{l}\text { Sé cómo relajarme y controlar mis } \\
\text { emociones }\end{array}$} & Padre & $2.84(.74)$ & \multirow{2}{*}{4.911} & \multirow{2}{*}{.000} & \multirow{2}{*}{.26} \\
\hline & & Madre & $2.65(.69)$ & & & \\
\hline & \multirow{2}{*}{$\begin{array}{l}\text { En el día a día me relajo y controlo } \\
\text { cuando estoy alterado/a }\end{array}$} & Padre & $2.83(.74)$ & \multirow{2}{*}{5.025} & \multirow{2}{*}{.000} & \multirow{2}{*}{.25} \\
\hline & & Madre & $2.65(.67)$ & & & \\
\hline & \multirow{2}{*}{$\begin{array}{l}\text { Sé relajarme y controlar mis emocio- } \\
\text { nes ante mis hijos/as }\end{array}$} & Padre & $2.75(.79)$ & \multirow{2}{*}{2.016} & \multirow{2}{*}{.044} & \multirow{2}{*}{.10} \\
\hline & & Madre & $2.67(.74)$ & & & \\
\hline \multirow{6}{*}{$\begin{array}{l}\text { F4- } \\
\text { Comunicación }\end{array}$} & \multirow{2}{*}{$\begin{array}{l}\text { Les digo a mis hijos/as cómo me } \\
\text { siento con su manera de actuar }\end{array}$} & Padre & $2.99(.91)$ & \multirow{2}{*}{-7.061} & \multirow{2}{*}{.000} & \multirow{2}{*}{-.37} \\
\hline & & Madre & $3.31(.79)$ & & & \\
\hline & \multirow{2}{*}{$\begin{array}{l}\text { En el día a día suelo decirles a mis } \\
\text { hijos/as lo positivo que veo en ellos/ } \\
\text { as }\end{array}$} & Padre & $2.82(.84)$ & \multirow[b]{2}{*}{-5.636} & \multirow[b]{2}{*}{.000} & \multirow[b]{2}{*}{-.3} \\
\hline & & Madre & $3.07(.83)$ & & & \\
\hline & \multirow{2}{*}{$\begin{array}{l}\text { Hago ver a mis hijos/as que son ca- } \\
\text { paces de tomar decisiones aunque } \\
\text { sean pequeños/as }\end{array}$} & Padre & $3.12(.80)$ & \multirow{2}{*}{-3.939} & \multirow[b]{2}{*}{.000} & \multirow[b]{2}{*}{-.2} \\
\hline & & Madre & $3.29(.79)$ & & & \\
\hline
\end{tabular}

Competencias parentales diferenciales del padre y de la madre en función de la etapa escolar de sus adolescentes (ESO o Bachillerato/FP; fase inicial o tardía de la adolescencia)

El contraste de grupos identifica diferencias significativas solo en F3-Imposición, con tamaño del efecto medio $(p=.000, d=$
.22); más específicamente en dos indicadores (Tabla 2), también con tamaño del efecto medio. En ambos casos los progenitores con adolescentes en ESO (adolescencia inicial) tienden a imponerse más que quienes tienen adolescentes mayores que cursan Bachillerato/FP.

Tabla 2

Diferencias estadísticamente significativas en función de la etapa escolar de los hijos/as (ESO-B/FP)

\begin{tabular}{|c|c|c|c|c|c|c|}
\hline Factor & Indicador/Ítem & Etapa & $M(D T)$ & $t$ & $p$ & $d$ \\
\hline \multirow{4}{*}{ F3- Imposición } & \multirow{2}{*}{$\begin{array}{l}\text { Cuando mis hijos/as me desobedecen, lo mejor es } \\
\text { imponerse para solucionar la situación }\end{array}$} & ESO & $2.50(.92)$ & \multirow{2}{*}{3.793} & \multirow{2}{*}{.000} & \multirow{2}{*}{.22} \\
\hline & & $\mathrm{B} / \mathrm{FP}$ & $2.30(.91)$ & & & \\
\hline & \multirow{2}{*}{$\begin{array}{l}\text { Cuando mis hijos/as intentan salirse con la suya } \\
\text { para conseguir algo, yo me impongo más para con- } \\
\text { trolar la situación }\end{array}$} & $\mathrm{ESO}$ & $2.57(.85)$ & \multirow[b]{2}{*}{3.303} & \multirow[b]{2}{*}{.001} & \multirow[b]{2}{*}{.19} \\
\hline & & $\mathrm{B} / \mathrm{FP}$ & $2.41(.83)$ & & & \\
\hline
\end{tabular}

Cuando el contraste se realiza por separado en madres y en padres los resultados son parecidos, pero con matices diferenciadores (Tabla 3). Las madres, tanto con adolescentes en ESO como en Bachillerato/FP, muestran competencias similares en F1-Control-Relajación, F2-Autoestima y F4-Comunicación; sin embargo, son significativamente más impositivas (F3) con sus adolescentes en ESO que con los mayores (Bachillerato/FP), con tamaño del efecto bajo $(p=.001, d=-.18)$. Estas diferencias se concretan en los dos mismos indicadores y en la misma dirección que la comentada anteriormente para la muestra conjunta de padres y madres, ambos con tamaño del efecto medio (Tabla 3). Los padres, sin embargo, muestran un perfil más diferenciado (tabla 3 ), observándose que quienes tienen adolescentes en Bachillerato/ FP tienen más Autoestima (F2) y son menos Impositivos (F3) que quienes tienen adolescentes en ESO; no obstante, se Comunican (F4) menos con los mayores (B/FP) que con los de ESO cuando se trata de decirles cosas positivas.

\section{Competencias parentales diferenciales del padre y de la madre en función de su nivel de estudios}

Los resultados del contraste de grupos indican diferencias estadísticamente significativas en F3-Imposición $\left(p=.000, \mathrm{~N}^{2}=.031\right)$, con tamaño del efecto bajo: los progenitores con nivel educativo más bajo se imponen más que quienes tienen estudios secundarios o universitarios. Se confirman estas diferencias en todos los indicadores de este factor F3-Imposición (Tabla 4). También se identifican diferencias significativas en indicadores de los restantes factores: 1) "Sé cómo relajarme y controlar mis emociones" (F1-Control-Relajación) con puntuaciones significativamente más altas entre progenitores universitarios, que entre quienes tienen estudios obligatorios $\left.\left(p=.015 ; \mathrm{N}^{2}=.006\right) ; 2\right)$ "Creo que soy un buen padre/madre" (F2-Autoestima): aquellos con estudios obligatorios puntúan significativamente más que los restantes ( $p=$ .002; $\left.\mathrm{N}^{2}=.009\right)$; 3 ) "En el día a día suelo decir a mis hijos/as lo positivo que veo en ellos/as" ( $\left.p=.047 ; \mathrm{N}^{2}=.004\right)$, y "Hago ver a mis hijos/as que son capaces de tomar decisiones" (F4-Comunicación; $\left.p=.022 ; \mathrm{N}^{2}=.005\right)$ : se observan puntuaciones significativamente más altas en ambos indicadores entre progenitores universitarios que entre quienes sólo tienen estudios obligatorios. 
Tabla 3

Diferencias estadísticamente significativas en función de la etapa escolar de los hijos/as (ESO-B/FP)

\begin{tabular}{|c|c|c|c|c|c|c|c|}
\hline Progenitor & Factor & Indicador/Ítem & Etapa & $M(D T)$ & $t$ & $p$ & $d$ \\
\hline \multirow{4}{*}{ MADRE } & \multirow{4}{*}{ F3- Imposición } & \multirow{2}{*}{$\begin{array}{l}\text { Cuando mis hijos/as me desobedecen, lo mejor } \\
\text { es imponerse para solucionar la situación }\end{array}$} & ESO & $2.47(.94)$ & \multirow{2}{*}{3.181} & \multirow{2}{*}{.001} & \multirow{2}{*}{.24} \\
\hline & & & $\mathrm{B} / \mathrm{FP}$ & $2.24(.92)$ & & & \\
\hline & & Cuando mis hijos/as intentan salirse con la & ESO & $2.61(.87)$ & \multirow[b]{2}{*}{3.166} & \multirow[b]{2}{*}{.002} & \multirow[b]{2}{*}{.24} \\
\hline & & $\begin{array}{l}\text { suya para conseguir algo, yo me impongo más } \\
\text { para controlar la situación }\end{array}$ & $\mathrm{B} / \mathrm{FP}$ & $2.40(.88)$ & & & \\
\hline \multirow{6}{*}{ PADRE } & \multirow{2}{*}{ F2- Autoestima } & \multirow{2}{*}{ Tengo buena opinión de mí mismo } & ESO & $3.02(.75)$ & \multirow{2}{*}{1.990} & \multirow{2}{*}{.047} & \multirow{2}{*}{.13} \\
\hline & & & $\mathrm{B} / \mathrm{FP}$ & $3.14(.74)$ & & & \\
\hline & \multirow{2}{*}{ F3- Imposición } & \multirow{2}{*}{$\begin{array}{l}\text { Cuando mis hijo/as me desobedecen, lo mejor } \\
\text { es imponerse para solucionar la situación }\end{array}$} & ESO & $2.52(.90)$ & \multirow{2}{*}{2.148} & \multirow{2}{*}{.031} & \multirow{2}{*}{.16} \\
\hline & & & $\mathrm{B} / \mathrm{FP}$ & $2.37(.91)$ & & & \\
\hline & \multirow{2}{*}{ F4- Comunicación } & \multirow{2}{*}{$\begin{array}{l}\text { En el día a día suelo decirles a mis hijos/as lo } \\
\text { positivo que veo en ellos/as }\end{array}$} & ESO & $2.89(.78)$ & \multirow{2}{*}{2.486} & \multirow{2}{*}{.013} & \multirow{2}{*}{.21} \\
\hline & & & $\mathrm{B} / \mathrm{FP}$ & $2.71(.93)$ & & & \\
\hline
\end{tabular}

Tabla 4

Diferencias significativas en función del nivel educativo de las madres y padres (obligatorios, secundarios y universitarios)

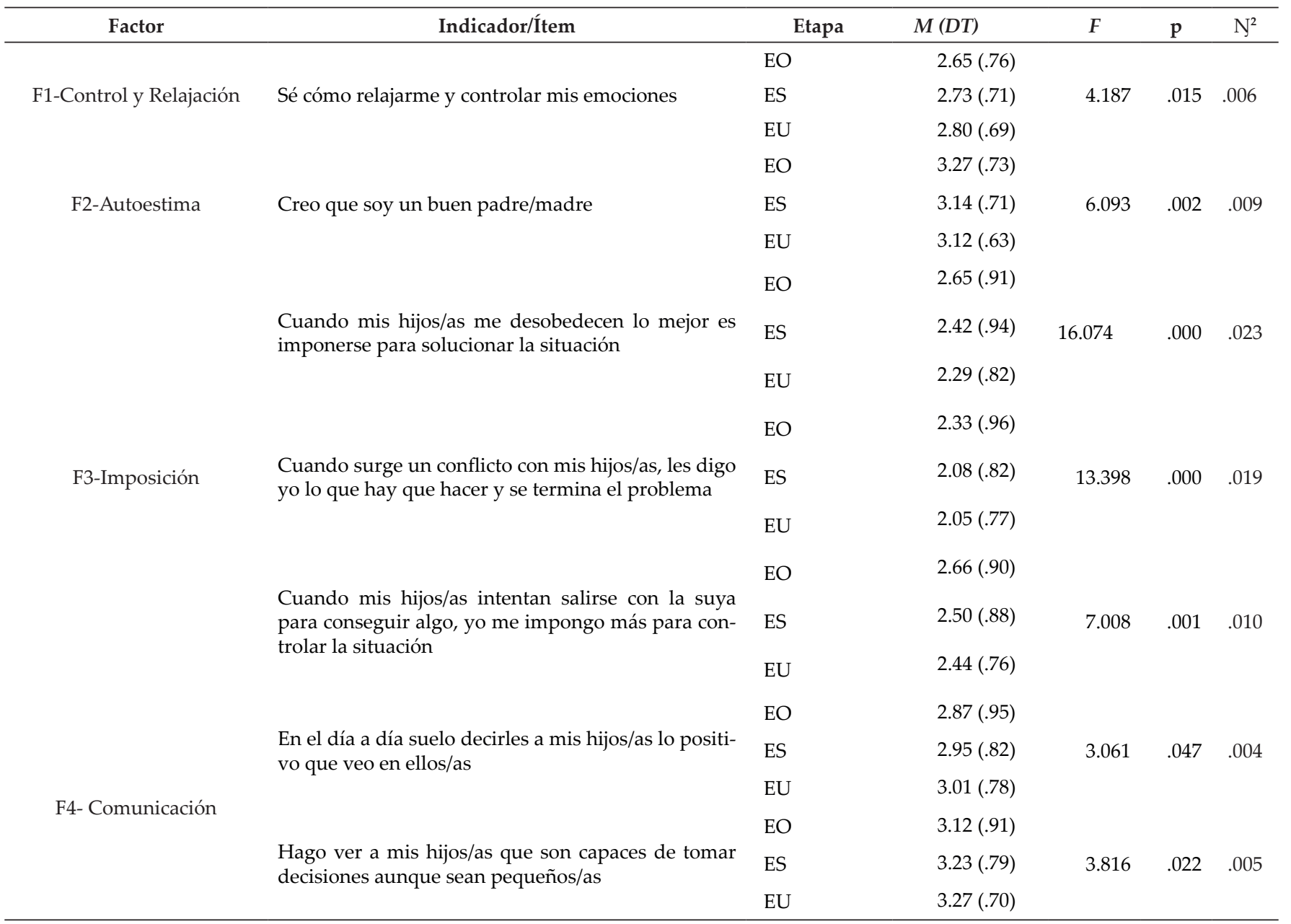

$\mathrm{EO}=$ Estudios Obligatorios; ES = Estudios Secundarios; EU = Estudios Universitarios 
Cuando el análisis se realiza por separado para madres y padres se confirman la mayor parte de los resultados mencionados, con algunos matices. En las madres se encuentran diferencias significativas en F3-Imposición, con tamaño del efecto bajo ( $p=$ $.000, \mathrm{~N}^{2}=.033$ ): aquellas con estudios obligatorios son significativamente más impositivas que quienes tienen estudios secundarios o universitarios; en concreto, las diferencias se advierten en los indicadores (Tabla 5): 1) “Cuando mis hijos/as me desobe- decen, lo mejor es imponerse para solucionar la situación" ( $p=$ $\left..001 ; \mathrm{N}^{2}=.020\right)$ y 2) "Cuando surge un conflicto con mis hijos/as, les digo yo lo que hay que hacer y se termina el problema" ( $p=$ $\left..000 ; \mathrm{N}^{2}=.029\right)$, ambos con tamaño del efecto bajo. Así mismo, se obtienen diferencias significativas en el indicador de F2-Autoestima "Creo que soy una buena madre", indicando que aquellas con estudios obligatorios son quienes más autoestima muestran, con tamaño del efecto bajo $\left(p=.003 ; \mathrm{N}^{2}=.015\right)$.

Tabla 5

Diferencias estadísticamente significativas en la Madre y en el Padre en función de su nivel educativo (obligatorio, secundario y universitario)

\begin{tabular}{|c|c|c|c|c|c|c|c|}
\hline Progenitor & Factor & Indicador/Ítem & Etapa & M (DT) & $F$ & p & $\mathrm{N}^{2}$ \\
\hline \multirow{9}{*}{ MADRE } & \multirow{3}{*}{ F2. Autoestima } & \multirow{3}{*}{ Creo que soy una buena madre } & $\mathrm{EO}$ & $3.35(.70)$ & \multirow{3}{*}{5.728} & \multirow{3}{*}{.003} & \multirow{3}{*}{.015} \\
\hline & & & ES & $3.13(.71)$ & & & \\
\hline & & & EU & $3.17(.62)$ & & & \\
\hline & \multirow{6}{*}{ F3. Imposición } & \multirow{3}{*}{$\begin{array}{l}\text { Cuando mis hijos/as me desobedecen lo } \\
\text { mejor es imponerse para solucionar la } \\
\text { situación }\end{array}$} & $\mathrm{EO}$ & $2.62(1.02)$ & \multirow{3}{*}{7.503} & \multirow{3}{*}{.001} & \multirow{3}{*}{.02} \\
\hline & & & ES & $2.37(.98)$ & & & \\
\hline & & & EU & $2.28(.81)$ & & & \\
\hline & & \multirow{3}{*}{$\begin{array}{l}\text { Cuando surge un conflicto con mis hijos, } \\
\text { les digo yo lo que hay que hacer y se ter- } \\
\text { mina el problema }\end{array}$} & $\mathrm{EO}$ & $2.35(.96)$ & \multirow{3}{*}{11.193} & \multirow{3}{*}{.000} & \multirow{3}{*}{$.02 \mathrm{C}$} \\
\hline & & & ES & $2.02(.82)$ & & & \\
\hline & & & EU & $2.01(.73)$ & & & \\
\hline \multirow{12}{*}{ PADRE } & \multirow{3}{*}{$\begin{array}{l}\text { F1. Control y } \\
\text { Relajación }\end{array}$} & \multirow{3}{*}{$\begin{array}{l}\text { Sé cómo relajarme y controlar mis emo- } \\
\text { ciones }\end{array}$} & $\mathrm{EO}$ & $2.72(.81)$ & \multirow{3}{*}{4.474} & \multirow{3}{*}{.012} & \multirow{3}{*}{.014} \\
\hline & & & ES & $2.81(.72)$ & & & \\
\hline & & & $\mathrm{EU}$ & $2.94(.70)$ & & & \\
\hline & \multirow{6}{*}{ F3. Imposición } & \multirow{3}{*}{$\begin{array}{l}\text { Cuando mis hijos/as me desobedecen lo } \\
\text { mejor es imponerse para solucionar la } \\
\text { situación }\end{array}$} & $\mathrm{EO}$ & $2.68(.96)$ & \multirow{3}{*}{8.375} & \multirow{3}{*}{.000} & \multirow{3}{*}{.025} \\
\hline & & & ES & $2.47(.90)$ & & & \\
\hline & & & EU & $2.31(.82)$ & & & \\
\hline & & \multirow{3}{*}{$\begin{array}{l}\text { Cuando mis hijos/as intentan salirse con } \\
\text { la suya para conseguir algo yo me im- } \\
\text { pongo más para controlar la situación }\end{array}$} & $\mathrm{EO}$ & $2.64(.89)$ & \multirow{3}{*}{4.806} & \multirow{3}{*}{.008} & \multirow{3}{*}{.015} \\
\hline & & & ES & $2.51(.85)$ & & & \\
\hline & & & EU & $2.38(.70)$ & & & \\
\hline & \multirow{3}{*}{ F4. Comunicación } & \multirow{3}{*}{$\begin{array}{l}\text { En el día a día suelo decirles a mis hijos/ } \\
\text { as lo positivo que veo en ellos/as }\end{array}$} & $\mathrm{EO}$ & $2.63(.95)$ & \multirow{3}{*}{5.806} & \multirow{3}{*}{.003} & \\
\hline & & & ES & $2.87(.82)$ & & & .017 \\
\hline & & & EU & $2.90(.75)$ & & & \\
\hline
\end{tabular}

$\mathrm{EO}=$ Estudios Obligatorios; ES = Estudios Secundarios; EU = Estudios Universitarios

En el caso de los padres se observan diferencias significativas en F3-Imposición, con tamaño del efecto bajo $\left(p=.000, \mathrm{~N}^{2}\right.$ $=.031$ ); más específicamente, en los indicadores (Tabla 5): 1) "Cuando mis hijos/as me desobedecen, lo mejor es imponerse para solucionar la situación" ( $\left.p=.000 ; \mathrm{N}^{2}=.025\right)$ y 2) "Cuando mis hijos/as intentan salirse con la suya para conseguir algo, yo me impongo más para controlar la situación" $\left(p=.008 ; \mathrm{N}^{2}=.015\right)$, indicando mayor actitud impositiva entre los padres con estudios obligatorios que entre los universitarios, ambos con tamaño del efecto bajo. También se encuentran diferencias significativas en F4-Comunicación, con tamaño del efecto bajo $\left(p=.007, \mathrm{~N}^{2}=\right.$ .015), confirmándose que los padres con estudios obligatorios se comunican peor con sus adolescentes que quienes tienen estudios secundarios; más en concreto, en el indicador "En el día a día suelo decirles a mis hijos/as lo positivo que veo en ellos/as", los padres con estudios obligatorios puntúan significativamente menos que los restantes ( $\left.p=.003 ; \mathrm{N}^{2}=.017\right)$. Por último, se han constatado diferencias significativas en el indicador "Sé cómo relajarme y controlar mis emociones" (F1-Control-Relajación; $p$ $=.012 ; \mathrm{N}^{2}=.014$ ), indicando mayor puntuación entre los universitarios que entre quienes tienen estudios obligatorios, con tamaño del efecto bajo. Por su parte, para concluir, el análisis de la interacción entre el género de los progenitores y su nivel de estudios resultó también significativo en todos los factores (F1-Control-Relajación, $p=.000$; F2-Autoestima, $p=.024$; F3-Imposición, $p=.000$; F4-Comunicación, $p=.000$ ), sugiriendo que las competencias parentales difieren al considerar conjuntamente ambas variables sociodemográficas, en el sentido que se ha indicado en los análisis previos (Tabla 6). 
Tabla 6

Diferencias significativas en los factores al considerar la interacción entre el género de los progenitores y su nivel educativo

\begin{tabular}{l|c|c|c}
\hline \multicolumn{1}{c|}{ Factor } & $F$ & $p$ & $R^{2}$ Ajustado \\
\hline F1- Control y Relajación & 4.910 & .000 & .014 \\
\hline F2- Autoestima & 2.588 & .024 & .006 \\
\hline F3-Imposición & 7.643 & .000 & .023 \\
\hline F4- Comunicación & 13.495 & .000 & .042 \\
\hline
\end{tabular}

\section{Discusión y Conclusiones}

La teoría DPA sostiene que ejercer con eficacia el rol parental con adolescentes contribuye a su desarrollo adaptativo en sus distintos contextos de interacción (Oliva et al., 2008; Rodríguez-Ruiz et al., 2015); para ello, los progenitores necesitan competencias parentales que les ayuden a relacionarse con sus hijos e hijas. La mayoría de los estudios realizados al respecto suelen considerar conjuntamente a ambos progenitores sin diferenciar el género y sin que necesariamente ambos pertenezcan a la misma unidad familiar (García-Linaresa et al., 2014), a pesar de que desde la Teoría del Rol de Género (Potuchek, 1990) se indique que ambos se implican de modo diferencial en la crianza y educación de sus hijos e hijas, especialmente en la etapa adolescente (Pleck, 1997), y que ello puede llevar asociadas distintas competencias parentales. Por otra parte, el nivel educativo de los progenitores y el momento inicial o avanzando de la adolescencia de sus hijos e hijas, pueden ejercer algunas variaciones en el modo de manifestar estas competencias. De ahí que el objetivo de este estudio haya sido identificar si en una muestra aleatoria de padres y madres que pertenecen a la misma unidad familiar existen estas diferencias al analizar su habilidad para regular sus propias emociones, para mantener su autoestima y asertividad, para comunicarse positivamente con sus hijos e hijas, y para evitar la imposición.

Los resultados obtenidos muestran que ambos progenitores tienden a valorarse como competentes en su rol parental (F2-Autoestima) y con habilidades comunicativas con sus adolescentes (F4-Comunicación). No obstante, se ha encontrado también en ambos cierta tendencia impositiva (F3-Imposición) y falta de habilidad de autorregulación emocional (F1-Control-Relajación). Estos aspectos han sido indicados también en otras investigaciones sobre prácticas parentales con adolescentes asociadas a estrés parental (Oliva et al., 2007; Rodríguez-Ruiz et al., 2015); señalando desde la teoría DPA que si estas prácticas son sistemáticas pueden limitar el desarrollo de la autonomía de los hijos e hijas, y condicionar esta competencia en la edad adulta; así mismo, pueden ocasionar distanciamiento emocional con los progenitores. Bonds et al. (2002) sostienen, además, que los progenitores con más estrés son quienes se perciben de manera más negativa. Respecto a diferencias de género entre padres y madres, los resultados indican que las madres se sinceran con sus adolescentes con más frecuencia que los padres, comentándoles más cómo se sienten con su manera de actuar y los aspectos positivos que ven en ellos y ellas (F4-Comunicación), al igual que señalan Sánchez-Núñez et al. (2008). Estos resultados indican mayor competencia comunicativa en las madres que en los padres para relacionarse con sus adolescentes, y para fomentar su autoestima. Esta diferencia quizás esté asociada a otro resultado diferencial obtenido en este estudio, relativo a que los padres perciben autocontrolarse mejor emocionalmente que las madres tanto a nivel general como ante sus hijos e hijas (F1-Control-Relajación), lo que les llevaría a no necesitar tanto expresarles cómo se sienten con su manera de actuar. Esta menor habilidad de autocontrol por parte de las madres quizás puede deberse a su mayor implicación emocional en la crianza y educación de los hijos e hijas, tal como han manifestado Laible y Caro (2004); así como a que asumen más tareas vinculadas con el funcionamiento del hogar (Meteyer y Perry-Jenkins, 2010). Esto les puede generar un elevado nivel de estrés, sobre todo si trabajan también fuera del hogar y tienen dificultad para conciliar su vida personal, familiar y laborar, limitándoles más que a los padres a la hora de gestionar adecuadamente sus emociones ante sus adolescentes.

La fase inicial o tardía de la adolescencia en que se encuentren los hijos e hijas, relacionados en este estudio con la etapa escolar de ESO o Bachiller/FP, respectivamente, también se asocia a diferencias en competencias parentales. Los resultados indican que ambos progenitores tienden a ser más impositivos con los más jóvenes, que se encuentran en la ESO, que con los mayores que cursan Bachiller/FP (Oliva et al., 2008). Quizás se deba a que al inicio de esta etapa los progenitores tienen más dificultad para entender el cambio evolutivo y el modo a veces retador de comportarse sus hijos e hijas. Considerando diferencias de género entre padres y madres, estas últimas muestran en este estudio niveles similares de autoestima y comunicación con sus adolescentes en ambas fases, mientras que los padres presentan más autoestima cuando interaccionan con sus adolescentes mayores que están en Bachiller/FP, y comentan menos aspectos positivos a los más jóvenes, que cursan ESO.

Respecto a la influencia del nivel de estudios de los progenitores en el desempeño de su rol parental con adolescentes, los resultados de este estudio señalan que quienes tienen titulación universitaria muestran mayor grado en las competencias parentales evaluadas que quienes tienen solo estudios obligatorios (Huang, 2018); a excepción de la autoestima, que se produce a la inversa. Resultados similares han sido señalados por Teti y Candelaria (2002), quienes lo relacionan con que los progenitores con estudios superiores tienen una mayor percepción de autoeficacia, influyendo positivamente en sus competencias parentales. En este estudio se obtienen también diferencias asociadas a la interacción entre el género y el nivel educativo de los progenitores; así, en los padres, aquellos con estudios superiores perciben que se autorregulan mejor y que transmiten aspectos más positivos a sus adolescentes que quienes tienen estudios obligatorios, tal como constatan también Martínez-González et al. (2007). Estos resultados no se han obtenido en las madres; sin embargo, aquellas con estudios solo obligatorios se autoperciben más positivas y con mejor autoestima en su rol educativo que quienes tienen estudios superiores, tal como también han señalado Rodríguez-Ruiz et al. (2015). Quizás estas últimas sean más exigentes consigo mismas y con sus adolescentes, y les genere mayor cuestionamiento de su función educativa (Tur-Porcar et al., 2012). Schmid et al. (2011) encontraron también mayor tendencia a la depresión en las madres con estudios universitarios en un estudio sobre estilos parentales y desarrollo positivo en adolescentes. 
Como implicaciones prácticas de este estudio cabe mencionar que la gestión de las emociones en el caso de las madres, y la comunicación afectiva, en especial en los padres, constituyen competencias relevantes a reforzar en programas parentales dirigidos a familias con adolescentes que inician esta etapa evolutiva; en esta fase inicial se han percibido mayores dificultades de relación con sus hijos e hijas (Oliva et al., 2008). Así mismo, los progenitores con niveles educativos más bajos, ya sean padres o madres, necesitan un mayor apoyo para modificar su tendencia impositiva y para desarrollar habilidades educativas basadas en la negociación, el afecto y la comunicación. Ambos progenitores podrían formar parte de un mismo programa grupal dirigido a fomentar sus competencias parentales, posibilitándoles contrastar y complementar sus respectivas autopercepciones como padres y madres, ambas relevantes. Lo mismo cabe decir con respecto a incluir en un mismo grupo de trabajo a progenitores con distintos niveles educativos y cuyos adolescentes se encuentren en distintas fases de la adolescencia; la metodología experiencial, participativa y cooperativa de estos programas facilita el aprendizaje mutuo entre los y las participantes para mejorar sus competencias parentales.

Para finalizar cabe indicar algunas potencialidades y limitaciones de este trabajo como futuras líneas de investigación. Entre las primeras se encuentra contar con una muestra representativa de progenitores que facilita generalizar en cierta medida los resultados obtenidos. Por otra parte, el hecho de que la mayoría de estos progenitores formen parte de la misma unidad familiar, aún poco frecuente en los estudios realizados, facilita contrastar mejor por género sus competencias parentales. Entre las limitaciones y posibles futuras investigaciones se encuentra contrastar los resultados de los progenitores con la perspectiva de sus adolescentes para obtener una visión más ajustada de las interacciones entre ambos y su posible impacto en el desarrollo positivo adolescente (DPA). Se podrían incorporar también datos cualitativos para enriquecer con argumentaciones y vivencias las tendencias estadísticas encontradas en este trabajo, en especial sobre actitudes impositivas y poco comunicativas. Así mismo, podrían realizarse estudios longitudinales para analizar variaciones en competencias parentales en función de las etapas evolutivas de los hijos e hijas, y sus efectos en su desarrollo personal. Esto permitiría ajustar mejor los objetivos de los programas parentales.

\section{Referencias}

Baumrind, D. (1991a). Parenting styles and adolescent development. En R. M., Lerner, A. C., Petersen, \& J. Brooks-Gunn, (Eds.). Encyclopedia of adolescence, 2. (pp. 746-758). Garland Publishing.

Baumrind, D. (1991b). The influence of parenting style on adolescent competence and substance use. Journal of Early Adolescence, 11, 56-95. https://doi.org/10.1177/0272431691111004

Belsky, J., Bell, B., Bradley, R. H., Stallard, N., \& Stewart-Brown, S. L. (2007). Socioeconomic risk, parenting during the preschool years and child health age 6 years. The European Journal of Public Health, 17(5), 508-513. https://doi.org/10.1093/eurpub/ckl261

Bøe, T., Sivertsen, B., Heiervang, E., Goodman, R., Lundervold, A. J., \& Hysing, M. (2014). Socioeconomic status and child mental health: The role of parental emotional wellbeing and parenting practices. Journal of Abnormal Child Psychology, 42(5), 705-715. https://doi.org/10.1007/s10802-013-9818-9.

Bonds, D. D., Gondoli, D. M., Sturge-Apple, M. L., \& Salem, L. N. (2002). Parenting stress as a mediator of the relation between parenting support and optimal parenting. Parenting:
Science and Practice, 2(4), 409-435. https://doi.org/10.1207/ S15327922PAR0204_04

Bronfenbrenner, U. (1987). La ecología del desarrollo humano. Paidós.

Browne, M. W., \& Cudeck, R. (1993). Alternative ways of assessing model fit. In K. A., Bollen, \& J. S. Long, (Eds.). Testing structural equation models (pp. 136-162). Sage

Cohen, J. (1973). Eta-squared and Partial Eta-squared in Communication Science. Human Communication Research, 28, 473-490. Sin doi

Cohen, J. (1988). Statistical power analysis for the behavioral science. Lawrence ErlbaumAssociates.

Fletcher, A. C., Steinberg, L., \& Sellers, E. B. (1999). Adolescents' well-being as a function of perceived interparental-consistency. Journal of Marriage and the Family, 61(3), 599-610. https:// doi.org/10.2307/353563

Garaigordobil, M. (2005). Conducta antisocial durante la adolescencia: correlatos socio- emocionales, predictores y diferencias de género. Psicología Conductual, 13(2),197-215. Sin doi

García-Linaresa, M. C., García-Moralb, A. T., \& Casanova-Ariasa, P. F. (2014). Prácticas educativas paternas que predicen la agresividad evaluada por distintos informantes. Revista Latinoamericana de Psicolgía, 46(3), 198-210. https://doi.org/10.1016/ S0120-0534(14)70023-8

Huang, C. (2018). Parenting styles and the development of non-cognitive skills among Chinese adolescents. Society, 38(6), 223-247. https://doi.org/10.1016/j.chieco.2020.101477

Kan, K., \& Tsai, W. D. (2005). Parenting practices and children's education outcomes. Economics of Education Review, 24(1), 2943. https://doi.org/10.1016/j.econedurev.2004.04.006

Kerr, M., \& Stattin, H. (2000). What parents know, how they know it, and several forms of adolescent adjustment: Further support for a reinterpretation of monitoring. Developmental Psychology, 36, 366-380. https://doi.org/10.1037//00121649.36.3.366

Laible, D. J., \& Caro, G. (2004). The differential relations of maternal and paternal support and control to adolescence social competence, self-worth, and sympathy. Journal of Adolescent Research, 19, 759-782. https://doi.org/10.1177/ 0743558403260094

Lerner, R. M., Dowling, E. M., \& Anderson, P. M. (2003). Positive Youth Development: Thriving as the Basis of Personhood and Civil Society. Applied Developmental Science, 7(3) 172-180. https://doi.org/10.1207/S1532480XADS0703_8

Lerner, R. M., Lerner, J. V., Almerigi, J., \& Theokas, C. (2005). Positive Youth Development: A view of the issues. Journal of Early Adolescence, 25, 10-16. https://doi. org/10.1177/0272431604273211

Maccoby, E. E., \& Martín, J. A. (1983). Socialization in the context of the family: Parent-child interaction. En E. M. Hetherington, \& P. H. Mussen (Eds.), Handbook of child psychology: Socialization, personality and social development, 4 (pp.1-101). Wiley.

McLoyd, V. C., \& Smith, J. (2002). Physical discipline and behavior problems in African American, European American, and Hispanic children: Emotional support as a moderator. Journal of Marriage and Family, 64(1), 40-53. https:/doi.or$\mathrm{g} / 10.1111 / \mathrm{j} .1741-3737.2002 .00040 . x$

Martínez-González, R. A. (2009). Programa-Guía para el Desarrollo de Competencias Emocionales, Educativas y Parentales. Ministerio Español de Sanidad y Política Social.

Martínez-González, R. A., Pérez Herrero, M. H. \& Álvarez, L. (2007). Estrategias para prevenir y afrontar conflictos en las relaciones familiares (padres e hijos). Ministerio Español de Trabajo y Asuntos Sociales. 
Masten, A. S., \& Curtis, W. J. (2000). Integrating competence and psychopathology: Pathways toward a comprehensive science of adaption in development. Development and Psychopatholo$g y, 12,529-550$. https://doi.org/10.1017/s095457940000314x

Meteyer, K., \& Perry-Jenkins, M. (2010). Father involvement among working-class, dual-earner couples. Fathering, 8(3), 379-403. https://doi.org/10.3149/fth.0803.379

Moon M., \& Hoffman, C. D. (2008). Mothers' and fathers' differential expectancies and behaviors: parent $x$ child gender effects. The Journal of Genetic Psychology. 169(3) 261-279. https://doi.org/10.3200/GNTP.169.3.261-280.

Oliva, A., Hernando, A., Parra, A., Pertegal, M. A., Ríos, M., \& Antolín, L. (2008). La promoción del desarrollo adolescente: recursos y estrategias de intervención. Consejería de Salud de Andalucía.

Oliva, A., Parra, A., Sánchez-Queija, I., \& López, F. (2007). Estilos educativos materno y paterno: evaluación y relación con el ajuste adolescente. Anales de Psicología, 23(1), 49-56. https:// doi.org/10.1037/10422-009

Pleck, J. H. (1997). Paternal involvement: Levels, sources, and consequences. In M. E. Lamb (Ed.), The role of the father in child development (pp. 66-103). Wiley.

Pons-Salvador, G., Cerezo, M. A., \& Bernabé, G. (2005). Cambio y estabilidad en los factores que afectan negativamente la parentalidad. Psicothema, 17(1), 31-36. Sin doi

Ponzetti, J. J. (2016). Evidence-based parenting education: A global perspective. Routledge.

Potuchek, J. L. (1990). Employed wives' orientations to breadwinning: A gender theory analysis. Journal of Marriage and the Family, 54, 548-558. Sin doi

Rodrigo, M. J., Máiquez Chaves, M. L., Martín Quintana, J. C., Byrne, S., \& Rodríguez-Ruiz, B. (Coords.). (2015). Manual práctico de parentalidad positiva. Síntesis.

Rodríguez-Ruiz, B., Rodrigo, M. J., \& Martínez-González, R. A. (2015). Cross-Contextual Variability in Parents' and Tutors' Conflict Resolution Styles and Positive Development. The Journal of Educational Research, 108(6), 480-491. http://dx.doi.or $\mathrm{g} / 10.1080 / 00220671.2014 .905764$

Ryan, R. M., \& Deci, E. L. (2017). Self-determination theory: Basic psychological needs in motivation, development, and wellness. Guilford Press.
Sánchez-Nuñez, M. T., Fernández-Berrocal, P., Montañés-Rodríguez, J., \& Latorre-Postigo, J. M. (2008). ¿Es la inteligencia emocional una cuestión de género? Socialización de las competencias emocionales en hombres y mujeres y sus implicaciones. Revista de Investigación Psicoeducativa, 6, 455-47. http:// dx.doi.org/10.25115/ejrep.v6i15.1287

Sanders, R. A. (2013). Adolescent psychosocial, social, and cognitive development. Pediatrics in Review, 34(8), 354-358. http:// dx.doi.org/10.1542/pir.34-8

Schmid, K. L., Phelps, E., Kiely, M. K., Napolitano, C. M., Boyd, M. J., \& Lerner, R. M. (2011). The role of adolescents' hopeful futures in predicting positive and negative developmental trajectories: Findings from the 4-H Study of Positive Youth Development. The Journal of Positive Psychology, 6(1) 45-56. http://dx.doi.org/10.1080/17439760.2010.536777.

Sullivan, T. N., Kung, E. M., \& Farrell, A. D. (2004). Relation between witnessing violence and drug use initiation among rural adolescents: Parental monitoring and family support as protective factors. Journal of Clinical Child and Adolescent Psychology, 33(3) 488-98. http://dx.doi.org/10.1207/s15374424jccp3303_6.

Teti, D. M., \& Candelaria, M. (2002). Parenting competence. In M. Bornstein, Handbook of parenting, Vol. 4, Applied Parenting (pp. 149-180). Lawrence Erlbaum Associates

Tur-Porcar, A., Mestre, V, Samper, P., \& Malonda, E. (2012). Crianza y agresividad de los menores: ¿Es diferente la influencia del padre y de la madre?. Psicothema, 24(2), 284-288. Sin doi

Wang, M. T., Dishion, T. J, Stormshak, E. A., \& Willett, J. B. (2011). Trajectories of family management practices and early adolescent behavioral outcomes. Developmental Psychology, 47, 1324-134. http://dx.doi.org/10.1037/a0024026

Waters, E., \& Sroufe, L. A. (1983). Social competence as a developmental construct. Developmental Review, 3, 79-97. https://doi. org/10.1016/0273-2297(83)90010-2

Yárnoz Yaben, S. (2006). ¿Seguimos descuidando a los padres? El papel del padre en la dinámica familiar y su influencia en el bienestar psíquico de sus componentes. Anales de Psicología, 22(2), 175-185. Sin doi 
